\title{
Pregnant patients diagnosed with COVID-19 simulate a clinical picture of preeclampsia
}

\author{
Sara L. Ayvar-Cavero ${ }^{1 *}$, Lizeth Aquino-Ruelas ${ }^{1}$, Miriam C. Perez-Díaz ${ }^{2}$, \\ José M. Zepeda-Torres ${ }^{1}$, Fanny Elizabeth Ancona-de la Rosa ${ }^{1}$, Eva Emilia Martinez-Guerrero ${ }^{1}$
}

${ }^{1}$ School of Medicine, Autonomous University of Guadalajara, Guadalajara, Jalisco, Mexico

${ }^{2}$ University of the Valley of Mexico, Guadalajara, Jalisco, México

Received: 05 January 2022

Accepted: 20 January 2022

*Correspondence:

Dr. Sara L. Ayvar-Cavero,

E-mail: sara.ayvar@edu.uag.mx

Copyright: (C) the author(s), publisher and licensee Medip Academy. This is an open-access article distributed under the terms of the Creative Commons Attribution Non-Commercial License, which permits unrestricted non-commercial use, distribution, and reproduction in any medium, provided the original work is properly cited.

\section{ABSTRACT}

The current pandemic caused by the coronavirus 2019 (COVID-19) has been a relevant event; we have seen how it spreads rapidly and has an increased risk of becoming severe if the patient has any comorbidity. It is essential to consider that although COVID-19 is a respiratory disease, it can have systemic effects that can trigger various complications. This study is focused on how a COVID-19 infection can present symptoms similar to preeclampsia.

Keywords: COVID-19, Preeclampsia/HELLP, ACE2

\section{INTRODUCTION}

This review aims to compare SARS-Cov-2 infection and pre-eclampsia and to know the pathophysiological bases by which COVID-19 can induce a syndrome similar to preeclampsia.

Coronavirus disease 2019 (COVID-19) is a respiratory infection caused by coronavirus 2 (SARS-CoV-2), a newly discovered severe acute respiratory syndrome, which emerged in Wuhan, China, in December 2019. ${ }^{1}$

Coronavirus $(\mathrm{CoV})$ is a ribonucleic acid (RNA) virus of the Coronaviridae family, which causes respiratory and gastrointestinal infections ranging from mild, self-limiting conditions to severe disorders such as viral pneumonia with systemic involvement. ${ }^{1}$

The rapid spread of the disease prompted the world health organization, on January 30, 2020, to declare it a health emergency of international concern, based on the impact the virus could have on underdeveloped countries with less developed health infrastructures, and to recognize it as a pandemic on March 11. As of April 10, 2020, 182 countries reported positive cases of COVID-19, with 1,56,3857 confirmed cases and 95,044 deaths for a case fatality rate of $6.08 \% .^{2}$

According to the Pan American health organization, cases of COVID-19 among pregnant women now number more than 60,000 in the Americas. Some 458 of these women have died, mainly in Mexico, recorded 140 deaths and 5574 cases among pregnant and postpartum women. ${ }^{3}$ Therefore, it is of utmost importance to highlight those pregnant patients are part of one of the main risk groups for respiratory infections due to the physiological changes that occur during gestation, such as increased secretions and congestion in the upper respiratory tract, increased circumference of the chest wall and upward displacement of the diaphragm. ${ }^{3}$ These changes result in decreased residual volume, increased tidal volume and air trapping, slightly decreased airway, stable diffusing capacity, increased minute ventilation, and increased carbon dioxide chemosensitivity. ${ }^{3}$

While hypertensive disorders of pregnancy represent the most common complication during pregnancy, they affect $15 \%$ of pregnancies. They account for $18 \%$ of all maternal deaths worldwide, with an estimated 62,000 to 77,000 
deaths per year. They affect $15 \%$ of pregnancies and account for $18 \%$ of all maternal deaths worldwide, with an estimated 62,000 to 77,000 deaths per year. ${ }^{4}$ Three metaanalyses have compared the risk of adverse maternal and perinatal outcomes among pregnant women with and without SARS-CoV-2 infection. ${ }^{4-6}$ The results of these studies indicate that pregnant women with SARS-CoV-2 disease have a significantly increased risk of maternal death, ICU admission, preterm delivery, and fetal death. Also, infants born to mothers with SARS-CoV-2 infection were more likely to be admitted to the neonatal ICU. ${ }^{7}$

\section{LITERATURE REVIEW}

To develop this literature review, an exhaustive search of multiple scientific journals on the relationship between preeclampsia and SARS-Cov-2 infection was also conducted to investigate the development of a preeclampsia-like syndrome induced by SARS-Cov-2 infection.

\section{THEORETICAL FRAMEWORK}

Preeclampsia is a multisystem disorder whose clinical criteria are as follows: gestational age greater than 20 weeks, systolic blood pressure of $140 \mathrm{mmHg}$ or more or diastolic blood pressure of $90 \mathrm{mmHg}$ or more on two occasions at least 4 hours. ${ }^{8}$ However, women with gestational hypertension with severe range blood pressures (systolic blood pressure of $160 \mathrm{mmHg}$ or higher, or diastolic blood pressure of $110 \mathrm{mmHg}$ or higher) should be diagnosed with preeclampsia with severe features. ${ }^{8}$ Apart dipstick with 1+ or isolated urine sample with $30 \mathrm{mg}$ of protein in two samples within 4 to 6 hours of each other, and a urine dipstick with $1+$ or isolated urine sample with $30 \mathrm{mg}$ of protein in two samples within 4 to 6 hours of each other, and a urine dipstick with $1+$ or isolated urine sample with $30 \mathrm{mg}$ of protein in two samples within 4 to 6 hours of each other. ${ }^{8}$ In the absence of proteinuria, the diagnosis of preeclampsia may be established when gestational hypertension is associated with a criterion of severity, including persistent cerebral symptoms, visual disturbances, chest pain, dyspnea, epigastralgia or right upper quadrant pain with nausea or vomiting, signs of organ dysfunction or thrombocytopenia with alterations in liver enzyme concentrations. ${ }^{8}$ HELLP syndrome is usually considered a variant of PE. Diagnostic criteria are hemolysis with increased LDH $(>600 \mathrm{U} / 1)$ and AST $(\geq 70$ $\mathrm{U} / \mathrm{l})$, and platelets $<100000 / \mu \mathrm{l}^{10}$

COVID-19 during pregnancy and preeclampsia are strongly associated, especially among nulliparous women, because of vascular conditions preceding infection. ${ }^{1,8}$

A prospective observational study with 42 SARS-CoV-2 positive cases shows 34 cases with non-severe pneumonia and eight severe COVID. Of the eight, five pregnant COVID-19 women (62.5\%) develop features of PE; however, they only appeared in COVID19 cases complicated by severe pneumonia, with PE/HELLP diagnostic criteria. ${ }^{10}$

The importance of this lies in the fact that women during pregnancy are a group particularly susceptible to respiratory infections due to immunological changes that increase susceptibility to pathogens, among which is the decrease in chemotaxis, adhesion, and manufacture of reactive oxygen products in neutrophils, also the peripheral B lymphocyte count decreases, especially in the first and third trimester of gestation, there is a decrease in the activation of complement to avoid a response against paternal antigens found on the surface of trophoblast cells. ${ }^{11}$ These multiple changes prevent rejection of the fetus and aid fetal development. Key cardiopulmonary adaptations of pregnancy include elevation of the diaphragm, increased oxygen consumption, and respiratory tract edema, making pregnant women more prone to hypoxia. ${ }^{12}$

The systematic review results by Di Mascio show that more than $90 \%$ of hospitalized pregnant women affected by SARS-CoV infections present radiological signs suggestive of pneumonia, detected on chest X-ray or CT scan, and the most common symptoms are fever, cough, and lymphopenia. ${ }^{1}$ Likewise, patients may develop hypertension, liver damage, renal failure, thrombocytopenia, among others, as a consequence of epithelial injury, thrombo-inflammation, and alterations in the angiotensin-converting enzyme produced by SARSCoV2. ${ }^{8}$

\section{INFECTION MECHANISM}

Among essential aspects that make pregnant women more susceptible to COVID-19 is that SARS-CoV-2 uses angiotensin-converting enzyme-converting protein (ACE2) as a receptor to invade cells, as shown in Figure $1 .{ }^{12}$

This analysis provides insight into the most critical components that explain how SARS-CoV2 infection during pregnancy could be involved in the pathogenesis of preeclampsia.

SARS-CoV-2 enters the cell after the N-terminal portion of the spike protein binds to the ACE2 receptor on the cell membrane.

The ACE2 receptor is a component of the RAS (Renin angiotensin aldosterone system), which converts angiotensin II to angiotensin 1 to 7.

The RAS is an essential regulator of placental function because it controls trophoblast proliferation, angiogenesis, and blood flow by maintaining the balance of its vasoconstrictor and vasodilator pathways facilitating fetal oxygenation in normal pregnancy. 
The binding of SARS-CoV-2 to ACE2 receptors causes downregulation of the RAS system with reduced levels of vasodilator angiotensin, thus leaving vasoconstrictor and proinflammatory effects of angiotensin II unopposed. ${ }^{12}$

On the other hand, soluble FMS-like tyrosine kinase (sFlt1) is associated with placental hypoxia, endothelial damage, sepsis, and acute lung injury, and an increase in this protein has been observed in patients infected with COVID-19. A study by Espino and Sosa evaluated the association between serum levels between RAS and sFlt-1 components in pregnant patients diagnosed with COVID19, where it was shown that in pregnant women with severe COVID19 pneumonia, plasma levels of ANG-II are reduced, and plasma levels of sFlt-1 are increased, compared to those with the non-severe disease. ${ }^{13}$ Thus, it is assumed that an imbalance between (sFlt-1) characterizes the primary component of endothelial dysfunction as an angiogenic marker and RAS components (ANG-II and ACE-2) that may be related to adverse outcomes in pregnant women diagnosed with COVID-19.

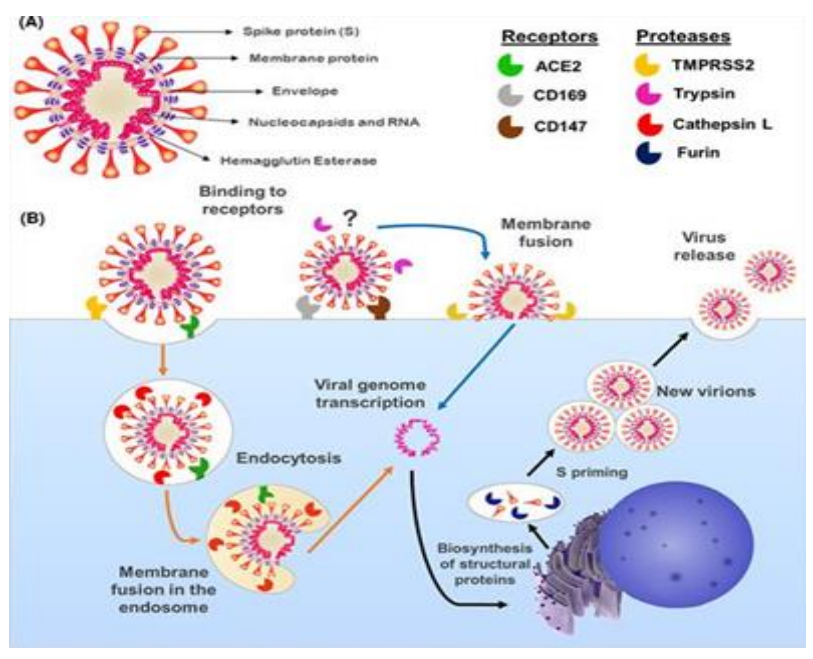

Figure 1: Viral replication (Azinheira et al 2021). ${ }^{11}$

\section{PLACENTAL ALTERATIONS}

Recall that syncytiotrophoblasts (STS) coordinate an immune response to infection and serve as a physical barrier to virus passage, demonstrating a higher level of VEGF expression mainly in the STS of terminal villi in parturient women with moderate COVID-19 to a greater extent than in women with mild disease severity. ${ }^{14}$ Also, transplacental passage of the virus usually increases with advancing gestational age. ${ }^{15}$ At the same time, the severity of the fetal injury is often variable, including bronchopulmonary dysplasia, hypoxic-ischemic encephalopathy, sepsis, anemia requiring transfusion, patent ductus arteriosus are requiring treatment or surgery, intraventricular hemorrhage, necrotizing enterocolitis, or retinopathy of prematurity diagnosed before hospital discharge; or admission to the neonatal intensive care unit for $\geq 7$ days; or neonatal death before hospital discharge. ${ }^{8}$
SARS-CoV-2 can infect the syncytiotrophoblast (STS) and activate inflammatory responses in placentas from women with a positive RT-PCR test result. ${ }^{16}$ Furthermore, immunohistochemistry, in situ hybridization, and RNA scoping are proposed to identify and confirm the presence of SARS-CoV-2 in fetal placental cells, mainly in STS and Hoffbauer cells. ${ }^{15}$

SARS-CoV-2 viral particles have been identified in the STS layer, placental cotyledon, placental submembrane, and placental intervillous inflammatory infiltrates with neutrophils, monocytes, and macrophages expressing activation markers. ${ }^{11}$

Previous studies have shown that trophoblast-derived extracellular vesicles harbour a unique microRNA (miRNA) cluster, expressed from the miRNA cluster on chromosome 19, that confers viral resistance to recipient cells, suggesting a paracrine function that enables the communication between placental cells to regulate their immunity to viral infections. ${ }^{15}$ Together with an increased number of syncytial nodes, these changes also indicate the development of placental hypoxia in parturient women with COVID-19. ${ }^{17}$ Thus among the placental abnormalities that have been described in pregnant women infected with RAS-CoV2 include diffuse peri valent fibrin, fetal vascular poor perfusion as evidenced by thrombi in fetal vessels, choriohemangioma, maternal vascular poor perfusion, and multifocal infarcts.

\section{DISCUSSION}

Starting with symptomatic COVID-19 patients, who can be identified with the development of radiological signs and symptoms associated with pneumonia (fever, cough, and lymphopenia), together with data on hypertension, liver damage, renal failure, thrombocytopenia; as a result of epithelial injury, thrombo-inflammation and alterations in the angiotensin-converting enzyme produced by SARSCoV-2, showing in Figure 2.

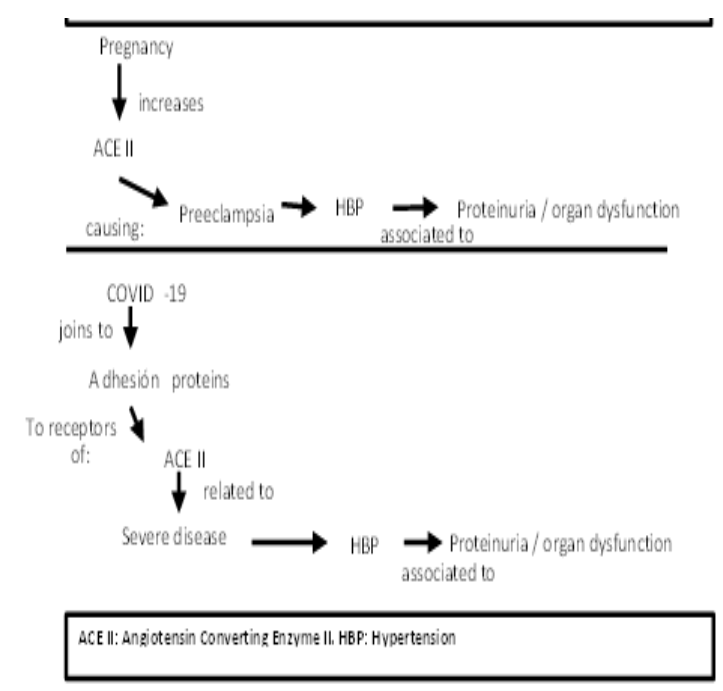

Figure 2: Pathological mechanism of both diseases. 
The same enzyme that we can find altered in preeclampsia. To reach this diagnosis, we know that for PE, we will discover new-onset arterial hypertension (blood pressure $>140 / 90 \mathrm{mmHg}$ ) usually after 20 weeks of gestation, which may be associated with proteinuria $(24 \mathrm{~h}$ urinary protein $>300 \mathrm{mg}$ or protein/creatinine ratio $\geq 0.3)$. Also, they can present organ dysfunction ${ }^{18}$ which we can also identify in infection caused by COVID-19 in pregnant women.

Of which are identified more markedly in nulliparous patients, with no significant difference if they were symptomatic of atypical pneumonia or not, including the fact of being diagnosed more than seven days after delivery with COVID-19 did not keep this relationship of both pathologies, and the weeks where you can see a higher prevalence is between 33 and $37 .{ }^{8}$ It is known that pregnancy complications are multiple, and the patient's prognosis depends on different factors. However, due to current health issues, infected pregnant women have been associated with a higher rate of preeclampsia than those classified as asymptomatic or not infected. ${ }^{8}$

When reviewing different articles, it is possible to identify that a very relevant factor in terms of risk of symptoms like preeclampsia is the infection of COVID-19, even among groups of the same age. ${ }^{16}$

Although the current evidence is limited, largely because it is a disease that we have only recently become aware of, it is not possible to ignore the potential risk of this pathology for the pregnant patient and the fetus, especially in light of some recent publications describing a generalized inflammatory reaction associated with a storm of cytokines and pro-inflammatory substances in patients with COVID-19.19 To differentiate between recently induced COVID19 hypertension, which is likely to be transient, and PE, specific molecular markers for preeclampsia, such as angiogenic factors, are needed. Like PIGF, sFlt-1, LDH y UtAPI. ${ }^{10,20}$ Identifying an imbalance of sFlt-1/PlGF is detectable in the maternal circulation at least five weeks before the onset of clinical PE. Patients with COVID-19 with a typical early stage of placental implantation should have normal sFlt-1/PIGF and UtAPI values despite proteinuria, thrombocytopenia, elevated liver enzymes, or hypertension. ${ }^{10}$

\section{CONCLUSSION}

The association of COVID-19 infection with pregnant patients represents a diagnostic challenge. They present thrombocytopenia and can show alterations in liver function, which are considered complications and criteria for preeclampsia with severe data. Or a relationship between this syndrome with the aggravation of COVID-19 infection. Likewise, an association has been found between COVID-19 and the development of a syndrome similar to preeclampsia/HELLP due to the pathogenesis of the same and the virus sharing a common pathway, using receptor binding (in the case of SARS-CoV-2) or the increase during pregnancy.

The relevancy of a good diagnosis which findings suggest that signs and symptoms compatible with COVID-19 and PE/HELLP too, could derive from the complex polypharmacy that we need to employ in patients at the moment of the diagnosis or from renal and cardiovascular dysfunction due to severe SARSCoV-2 infection that indicates the importance that can be differentiating between them on time.

Funding: No funding sources

Conflict of interest: None declared

Ethical approval: Not required

\section{REFERENCES}

1. Di Mascio D, Khalil A, Saccone G, Rizzo G, Buca D, Liberati $\mathrm{M}$ et al. Outcome of coronavirus spectrum infections (SARS, MERS, COVID-19) during pregnancy: a systematic review and meta-analysis. Am J Obstet Gynecol. 2020;2(2):100107.

2. Perlman S, Mclntosh. Coronaviruses, Including Severe Acute Respiratory Syndrome (SARS) and Middle East Respiratory Syndrome (MERS), Mandell, Douglas and Bennett's Principles and Practice of Infectious Diseases. 2020.

3. Narang K, Enninga E, Gunaratne M, Ibirogba ER, Trad A, Elrefaei A et al. SARSCoV-2 Infection and COVID-19 During Pregnancy: A Multidisciplinary Review. Mayo Clin Proc. 2020;95(8):1750-65.

4. Huntley B, Mulder IA, Di Mascio D, Vintzileos WS, Vintzileos AM, Berghella V et al. Adverse Pregnancy Outcomes Among Individuals with and without Severe Acute Respiratory Syndrome Coronavirus 2 (SARS-CoV-2): A Systematic Review and Metaanalysis. Obstet Gynecol, 2021;137(4):585-96.

5. Allotey J, Stallings E, Bonet M, Yap M, Chatterjee S, Kew $\mathrm{T}$ et al. Clinical manifestations, risk factors, and maternal and perinatal outcomes of coronavirus disease 2019 in pregnancy: living systematic review and meta-analysis. BMJ. 2019;370:m3320.

6. Wei SQ, Bilodeau-Bertrand M, Liu S, Auger N. The impact of COVID19 on pregnancy outcomes: a systematic review and meta-analysis. CMAJ. 2021;193(16):E540-8.

7. Montelongo FJ, Tapia-Velasco R, Salazar-Mendoza A, Suárez-Suárez A. Pre-eclampsia, eclampsia and impending eclampsia and their treatment. Therapeutics in Critical Medicine, McGraw Hill Medical. 2020.

8. Papageorghiou AT, Deruelle P, Gunier RB, Rauch S, García-May PK, Mhatre M, et al. Preeclampsia and COVID-19: results from the INTERCOVID prospective longitudinal study. Am J Obstet Gynecol. 2021;225(3):289.e1-17.

9. Mendoza M, Garcia-Ruiz I, Maiz N, Rodo C, GarciaManau P, Serrano B et al. Pre-eclampsia-like syndrome induced by severe COVID-19: a 
prospective observational study. BJOG. 2020;127(11):1374-80.

10. Tatiana MC, Kenia MC, Yaite ZR. Inmunología del embarazo e infección por COVID-19: una revisión en el contexto actual. Rev Cubana Hematol Inmunol Hemoter. 2021;37:1304.

11. Azinheira Nobrega Cruz N, Stoll D, Casarini D, Bertagnolli $\mathrm{M}$. Role of ACE2 in pregnancy and potential implications for COVID-19 susceptibility. Clin Sci (Lond). 2021;135(15):1805-24.

12. Espino-y-Sosa S, Martinez-Portilla RJ, Torres-Torres J, Solis-Paredes JM, Estrada-Gutierrez G, HernandezPacheco JA et al. Novel Ratio Soluble Fms-like Tyrosine Kinase1/Angiotensin-II (sFlt-1/ANG-II) in Pregnant Women Is Associated with Critical Illness in COVID-19. Viruses. 2021;13(10):1906.

13. Shchegolev AI, Kulikova GV, Lyapin VM, Shmakov RG, Sukhikh GT. The Number of Syncytial Knots and VEGF Expression in Placental Villi in Parturient Woman with COVID-19 Depends on the Disease Severity. Bull Exp Biol Med. 2021;171(3):399-03.
14. Golden TN, Simmons RA. Maternal and neonatal response to COVID-19. Am J Physiol Endocrinol Metab. 2020;319(2):315-9.

15. Conde-Agudelo A, Romero R. SARS-CoV-2 infection during pregnancy and risk of preeclampsia: a systematic review and meta-analysis. Am J Obstet Gynecol. 2021;226(1):68-89.

16. Naidoo N, Moodley J, Naicker T. Maternal endothelial dysfunction in HIV-associated preeclampsia comorbid with COVID-19: a review. Hypertens Res. 2021;44(4):386-98.

17. Barbieri RL, Repke JT. Medical Disorders During Pregnancy, 20e McGraw Hill Medical. 2018.

18. Córdoba-Vives S, Fonseca-Peñaranda G. COVID-19 y Embarazo. Rev Med Cos Cen 2020;86(629):22-9.

19. Jayaram A, Buhimschi IA, Aldasoqi H, Hartwig J, Owens T, Elam GL et al. Who said differentiating preeclampsia from COVID-19 infection was easy? Pregnancy Hypertens; 2021;26:8-10.

Cite this article as: Ayvar-Cavero SL, AquinoRuelas L, Perez-Díaz MC, Zepeda-Torres JM, Ancona-de la Rosa FM, Martinez-Guerrero EM. Pregnant patients diagnosed with COVID-19 simulate a clinical picture of preeclampsia. Int J Reprod Contracept Obstet Gynecol 2022;11:639-43. 